\begin{tabular}{|c|l|}
\hline Title & Similarity between nuclear rainbow and meteorological rainbow: Evidence for nuclear ripples \\
\hline Author(s) & Ohkubo, S.; Hirabay ashi, Y. \\
\hline Citation & $\begin{array}{l}\text { Physical Review C, 89(6), 61601 } \\
\text { https://doi.org/L0.1103/PhysRevC.89.061601 }\end{array}$ \\
\hline Issue Date & 201406-27 \\
\hline Doc URL & http://hdl.handle.net/2115/56861 \\
\hline Rights & @2014 A merican Physical Society \\
\hline Type & article \\
\hline File Information & PhysRevC.89.061601.pdf \\
\hline
\end{tabular}

Instructions for use 


\title{
Similarity between nuclear rainbow and meteorological rainbow: Evidence for nuclear ripples
}

\author{
S. Ohkubo ${ }^{1, *}$ and Y. Hirabayashi ${ }^{2}$ \\ ${ }^{1}$ Research Center for Nuclear Physics, Osaka University, Ibaraki, Osaka 567-0047, Japan \\ ${ }^{2}$ Information Initiative Center, Hokkaido University, Sapporo 060-0811, Japan \\ (Received 14 May 2014; revised manuscript received 10 June 2014; published 27 June 2014)
}

\begin{abstract}
We present evidence for the nuclear ripples superimposed on the Airy structure of the nuclear rainbow, which is similar to the meteorological rainbow. The mechanism of the nuclear ripples is also similar to that of the meteorological rainbow, which is caused by the interference between the externally reflective waves and refractive waves. The nuclear ripple structure was confirmed by analyzing the elastic angular distribution in ${ }^{16} \mathrm{O}+{ }^{12} \mathrm{C}$ rainbow scattering at $E_{L}=115.9 \mathrm{MeV}$ using the coupled channels method by taking account of coupling to the excited states of ${ }^{12} \mathrm{C}$ and ${ }^{16} \mathrm{O}$ with a double folding model derived from a density-dependent effective nucleon-nucleon force with realistic wave functions for ${ }^{12} \mathrm{C}$ and ${ }^{16} \mathrm{O}$. The coupling to the excited states plays the role of creating the external reflection.
\end{abstract}

DOI: 10.1103/PhysRevC.89.061601

PACS number(s): 25.70.Bc, 24.10.Eq, 42.25.Gy

Descartes [1] and subsequently Newton [2] explained the rainbow in optics by reflection and refraction in the raindrops. Airy [3] understood the supernumerary rainbow by the wave nature of light. The mechanism of the meteorological rainbow was understood precisely only recently by Nussenzveig using the electromagnetic theory of light [4]. In analogy with the meteorological rainbow the nuclear rainbow was predicted theoretically [5] and observed in $\alpha$ particle scattering [6]. The rainbow has been observed also in other systems such as in atom-atom collisions, atom-molecule collisions [7], electron-molecule collisions [8], and atom scattering from crystal surfaces [9]. Although the mechanism of Newton's zero-order ( $p=1$ in Fig. 1) nuclear rainbow [10], where only refraction is active, is very different from that of the meteorological rainbow ( $p=2$ in Fig. 1), a similar Airy structure has been observed. As shown in Fig. 2, the precise description of the meteorological rainbow given by solving Mie scattering shows the rapidly oscillating structure, the high-frequency ripple structure, superimposed on the Airy structure of the rainbow $[4,11,12]$. The ripple structure is not predicted by the semiclassical theory of the nuclear rainbow of Ref. [5] and no attention has been paid to its possible existence. Here we report for the first time evidence for the existence of the ripple structure in the observed nuclear rainbow and explain its mechanism.

The Airy structure of nuclear rainbows has been studied extensively especially for heavy ion scattering such as ${ }^{16} \mathrm{O}+{ }^{16} \mathrm{O},{ }^{16} \mathrm{O}+{ }^{12} \mathrm{C}$, and ${ }^{12} \mathrm{C}+{ }^{12} \mathrm{C}[13,14]$. For the most typical ${ }^{16} \mathrm{O}+{ }^{16} \mathrm{O}$ system, similar to the typical $\alpha+{ }^{16} \mathrm{O}$ and $\alpha+{ }^{40} \mathrm{Ca}$ scattering $[15,16]$, a global deep potential has been determined uniquely from the rainbow scattering. It reproduces the experimental data over a wide range of energies from negative energy to the incident energy $E_{L}=1120$ $\mathrm{MeV}$ - that is, the rainbows [17], prerainbows [18], molecular resonances and cluster structures with the superdeformed configuration [19] —in a unified way. Unfortunately the observed
Airy structure in the angular distributions is obscured due to symmetrization of two identical bosons.

In this respect rainbow scattering of the asymmetric ${ }^{16} \mathrm{O}+{ }^{12} \mathrm{C}$ system is important and has been thoroughly investigated [20-25]. A global deep potential could describe well the rainbows in the high-energy region, prerainbows [10], molecular resonances, and cluster structures with the ${ }^{16} \mathrm{O}+{ }^{12} \mathrm{C}$ configuration in the quasibound energy region in a unified way [26]. However at energies around $E_{L}=100 \mathrm{MeV}$ the global optical potential calculations [23] only reproduced the experimental angular distributions in a qualitative way at larger angles. Also, to reproduce the high-frequency oscillations, imaginary potentials-with a thin-skinned volume term and an extraordinary small diffuseness parameter around $0.1 \mathrm{fm}$ accompanying a surface term peaked at a larger radius-were needed $[23,25]$.

The purpose of this paper is to show that the high-frequency oscillations superimposed on the Airy structure are nothing but the ripple structure of the nuclear rainbow, and can be explained by fully taking account of coupling to the excited states of ${ }^{12} \mathrm{C}$ and ${ }^{16} \mathrm{O}$ by using the microscopic wave functions and the extended double folding model. The mechanism of the ripple structure and the role of coupling to the excited states is clarified, and the similarity between the macroscopic meteorological rainbow and the quantum nuclear rainbow, despite the difference of the underlying interactions, is discussed.

We study ${ }^{16} \mathrm{O}+{ }^{12} \mathrm{C}$ scattering with the coupled channels method using an extended double folding (EDF) model that describes all the diagonal and off-diagonal coupling potentials derived from the microscopic realistic wave functions for ${ }^{12} \mathrm{C}$ and ${ }^{16} \mathrm{O}$ using a density-dependent nucleon-nucleon force. The diagonal and coupling potentials for the ${ }^{16} \mathrm{O}+{ }^{12} \mathrm{C}$ system are calculated using the EDF model without introducing a normalization factor:

$$
\begin{aligned}
V_{i j, k l}(\mathbf{R})= & \int \rho_{i j}^{\left({ }^{16} \mathrm{O}\right)}\left(\mathbf{r}_{1}\right) \rho_{k l}^{\left({ }^{12} \mathrm{C}\right)}\left(\mathbf{r}_{2}\right) \\
& \times v_{N N}\left(E, \rho, \mathbf{r}_{1}+\mathbf{R}-\mathbf{r}_{2}\right) d \mathbf{r}_{1} d \mathbf{r}_{2}
\end{aligned}
$$




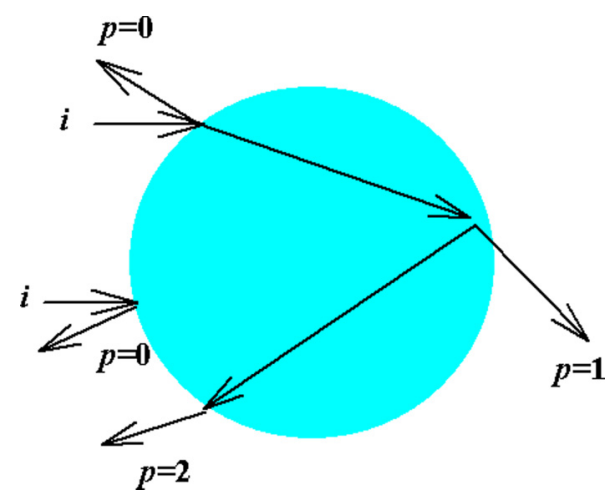

FIG. 1. (Color online) Illustrative figure of path of an incident ray $(i)$ in a spherical raindrop in geometrical optics. The rays $p=0$, $p=1$, and $p=2$ correspond to reflection, refraction only, and the primary rainbow, respectively.

where $\rho_{i j}^{(160)}(\mathbf{r})$ is the diagonal $(i=j)$ or transition $(i \neq j)$ nucleon density of ${ }^{16} \mathrm{O}$ taken from the microscopic $\alpha+{ }^{12} \mathrm{C}$ cluster model wave functions calculated in the orthogonality condition model (OCM) in Ref. [27]. This model uses a realistic size parameter both for the $\alpha$ particle and ${ }^{12} \mathrm{C}$, and is an extended version of the OCM $\alpha$ cluster model of Ref. [28], which reproduces almost all the energy levels well up to $E_{x} \approx 13 \mathrm{MeV}$ and the electric transition probabilities in ${ }^{16} \mathrm{O}$. We take into account the important transition densities available in Ref. [27], i.e., g.s. $\leftrightarrow 3^{-}(6.13 \mathrm{MeV})$ and $2^{+}(6.92 \mathrm{MeV})$ in addition to all the diagonal potentials. $\left.\rho_{k l}^{(12} \mathrm{C}\right)(\mathbf{r})$ represents the diagonal $(k=l)$ or transition $(k \neq l)$ nucleon density of ${ }^{12} \mathrm{C}$ calculated using the microscopic three- $\alpha$ cluster model in the resonating group method [29]. This model reproduces the structure of ${ }^{12} \mathrm{C}$ well, and the wave functions have been checked for many experimental data [29]. In the coupled channels calculations we take into account the $0_{1}^{+}(0.0 \mathrm{MeV}), 2^{+}(4.44 \mathrm{MeV})$, and $3^{-}$ $(9.64 \mathrm{MeV})$ states of ${ }^{12} \mathrm{C}$. The mutual excitation channels in which both ${ }^{12} \mathrm{C}$ and ${ }^{16} \mathrm{O}$ are excited simultaneously are not included. For the effective interaction $v_{\mathrm{NN}}$ we use the DDM3Y-FR interaction [30], which takes into account the

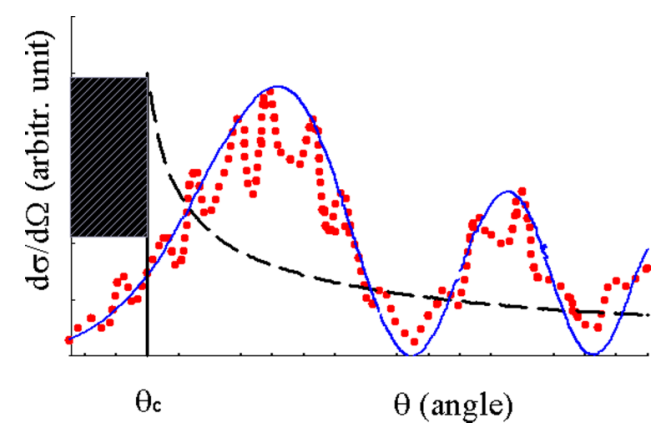

FIG. 2. (Color online) Cross sections for the primary rainbow in Fig. 1. The dashed line is by classical theory in optics and the dark region before the critical angle $\left(\theta_{c}=138^{\circ}\right)$ is displayed by a black shade. The dotted and solid lines show calculations of Mie scattering [11] and in the Airy approximation [11] in the wave theory of light, respectively.

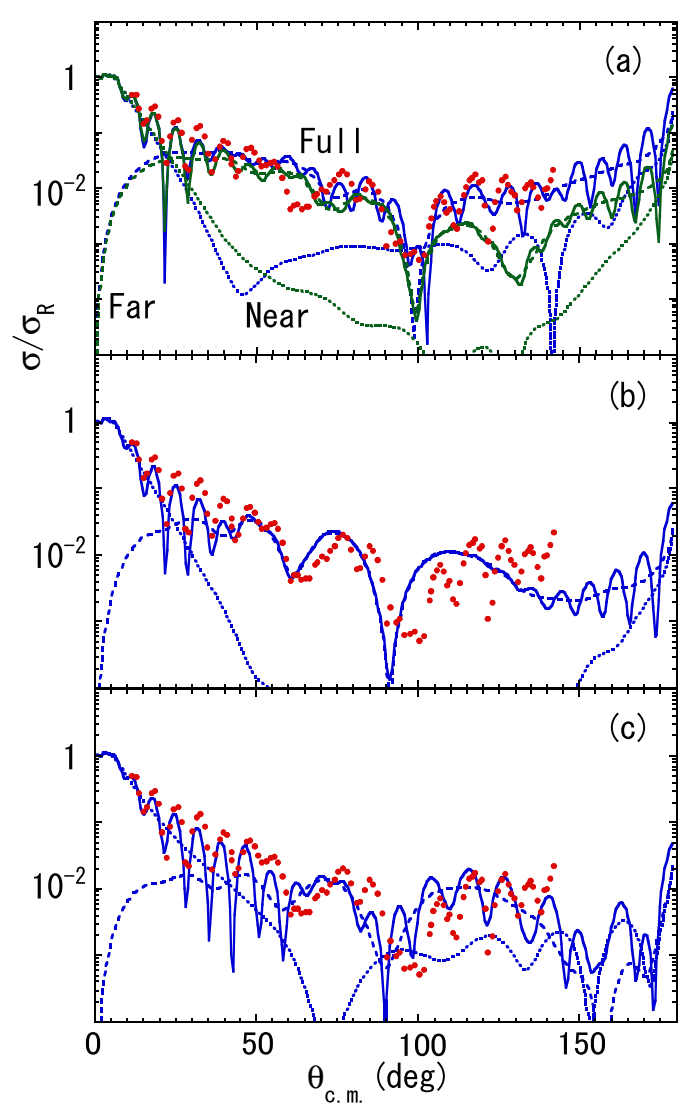

FIG. 3. (Color online) The experimental cross sections (points) in ${ }^{16} \mathrm{O}+{ }^{12} \mathrm{C}$ scattering at $E_{L}=115.9 \mathrm{MeV}$ [23] are compared with the calculations (solid line) using the EDF potential: (a) the coupled channels calculations with $a_{W}=0.2$ (blue line), (b) the single channel calculations with $a_{W}=0.6$, and (c) the single channel calculations using the extremely thin-skinned volume-type imaginary potential with $a_{W}=0.1$. For comparison, in (a) the coupled channels calculations with $a_{W}=0.4$ are displayed by the green line. The calculated cross sections (solid line) are decomposed into the farside (dashed line) and nearside (dotted line) components.

finite-range nucleon exchange effect. An imaginary potential (nondeformed) is introduced phenomenologically to take into account the effect of absorption due to other channels.

In Fig. 3(a) the angular distributions of elastic ${ }^{16} \mathrm{O}+{ }^{12} \mathrm{C}$ scattering at $E_{L}=115.9 \mathrm{MeV}$ calculated using the coupled channels method (blue solid line) are compared with the experimental data. We found that the EDF potential works well without introducing a normalization factor. The volume integral per nucleon pair of the ground state diagonal part, $J_{V}=317.7 \mathrm{MeV} \mathrm{fm}^{3}$, is consistent with those used in other optical potential model calculations and belongs to the same global potential family found in the $E_{L}=62-1503 \mathrm{MeV}$ region $[23,24,31]$. The parameters used in the imaginary potential with a Woods-Saxon volume-type form factor displayed in Fig. 4 are $W_{V}=14 \mathrm{MeV}, R_{W}=5.6 \mathrm{fm}$, and $a_{W}=0.20 \mathrm{fm}$ with a volume integral per nucleon pair $J_{W}=$ $54.3 \mathrm{MeV} \mathrm{fm}^{3}$, in the conventional notation. We see that the refractive farside scattering dominates at the intermediate and large angles. The calculation reproduces well the two 


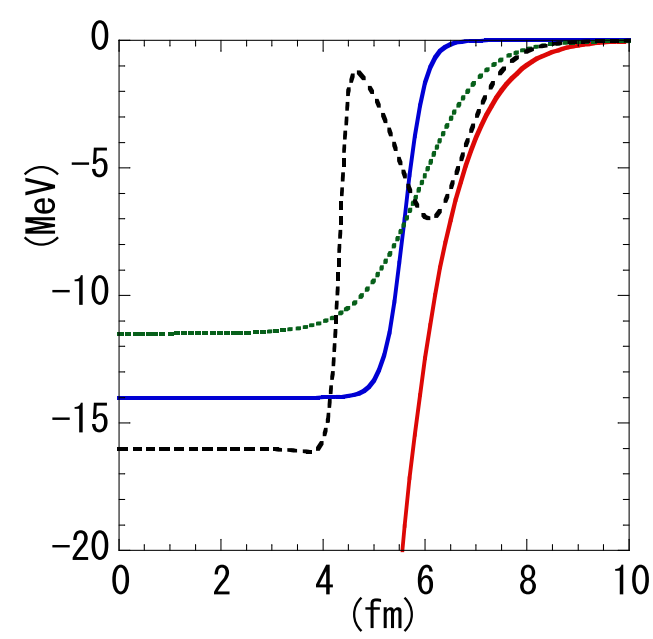

FIG. 4. (Color online) The real folding and Woods-Saxon volume-type imaginary potentials used in the coupled channels calculations [Fig. 3(a)] in ${ }^{16} \mathrm{O}+{ }^{12} \mathrm{C}$ scattering are displayed by the red solid line and the blue solid line, respectively. The imaginary potentials used in the single calculations in Figs. 3(b) and 3(c) are displayed by the green dotted line and the black dashed line, respectively.

broad Airy maxima in the angular range, $\theta=60-90^{\circ}$ (Airy maximum $A 2$ ) and $\theta=100-140^{\circ}$ (Airy maximum $A 1$ ) in the experimental angular distribution, which are brought about by the refracted farside component. Also the high-frequency oscillations superimposed on the two broad Airy maxima, A1 and $A 2$, in the experimental data are reproduced well. We see that the high-frequency oscillations are brought about by the interference between the farside and nearside scattering components. The investigation of the contributions of each channel reveals that none is overwhelmingly dominant and that the contribution of the excited states of ${ }^{16} \mathrm{O}$ is as much as that of ${ }^{12} \mathrm{C}$, which is quite different from the higher energy region around $E_{L}=300 \mathrm{MeV}$ where coupling to the $2^{+}$state of ${ }^{12} \mathrm{C}$ contributed dominantly in creating the secondary bow in the classically forbidden dark side of the primary rainbow [32]. Also neither the extremely thin-skinned volume-type imaginary potential with $a_{W}=0.1$ nor the surface imaginary potential peaked at a larger radius used in Refs. [23,25] were needed.

In Fig. 3(b) the angular distributions calculated in the single channel calculation using the readjusted imaginary potential (displayed in Fig. 4), $W_{V}=11.5 \mathrm{MeV}, R_{W}=5.9 \mathrm{fm}$, and $a_{W}=0.6 \mathrm{fm}\left(J_{W}=52.8 \mathrm{MeV} \mathrm{fm}{ }^{3}\right)$, which is similar to Ref. [31], are shown. Although the farside scattering is dominant, similar to Fig. 3(a), and the gross behavior of the Airy structure of the experimental angular distribution is reproduced, the high-frequency oscillations are missing. By comparing Fig. 3(a) (blue solid line) and Fig. 3(b), we note that the channel coupling to the excited states of ${ }^{16} \mathrm{O}$ and ${ }^{12} \mathrm{C}$ contributes in generating the high-frequency oscillations, although the rather small $a_{W}=0.2$ is needed. In Fig. 3(b) we note that the nearside component is damped more than two order of magnitude compared with the farside component, and no interference between them occurs resulting no highfrequency oscillations. While the $J_{W}$ values are almost the same for Figs. 3(a) and 3(b), the nearside scattering is retained significantly in Fig. 3(a). This means that the channel coupling in Fig. 3(a) plays a role of increasing the nearside scattering component, i.e., reflection.

This can be confirmed in Fig. 3(c) where the angular distributions calculated in the single channel using both the extremely thin-skinned volume-type (Woods-Saxon squared) imaginary potential with $W_{V}=16 \mathrm{MeV}, R_{W}=4.4 \mathrm{fm}$, and $a_{W}=0.1 \mathrm{fm}$ and the surface imaginary potential with $W_{D}=7 \mathrm{MeV}, R_{D}=6.1 \mathrm{fm}$, and $a_{D}=0.46 \mathrm{fm} \quad\left(J_{W}=\right.$ $59.8 \mathrm{MeV} \mathrm{fm}^{3}$ ) (displayed in Fig. 4), which are similar to those in Refs. [23] and [25], are shown. We note that the values of $J_{W}$ are almost the same for the three cases (a), (b), and (c). The high-frequency oscillations superimposed on the Airy structure are recovered only by using this extremely thin-skinned imaginary potential. We see that the nearside component needed to bring about the high-frequency oscillations is significantly increased compared with Fig. 3(b). The sharper the diffuseness of the imaginary potential is, the more the nearside component is increased. This can be checked by decreasing the ratio $a_{W} / W_{V}$; that is, if we increase the strength of the imaginary potential in Fig. 3(c) by $50 \%$ to $W_{V}=24 \mathrm{MeV}$ from the original $16 \mathrm{MeV}$, the magnitude of the calculated cross section and its farside component decrease as expected from the increase of absorption. However, the magnitude of the nearside component is increased. This means that for the nearside scattering the imaginary potential does not act as absorption but acts as "divergence," i.e., increasing reflective waves under the small diffuseness $a_{W}=0.1$. Thus the increased nearside component is found to be reflective in origin. The same behavior is observed for the coupled channels calculations in Fig. 3(a) with $a_{W}=0.2$. We see in Fig. 3(a) that the calculated cross sections with a moderate smooth diffuseness $a_{W}=0.4$ (green line) show no high-frequency oscillations, which means that no reflective waves are created. We note that the magnitudes of the $S$ matrix in Figs. 5(a) (blue line) and 5(a) (green line) are similar to those in Figs. 5(c) and 5(b), respectively.

We show that the high-frequency oscillations superimposed on the Airy structure in Fig. 3(a) are nothing but the ripple structure of the nuclear rainbow. In Fig. 2 the ripple structure in the meteorological rainbow is generated by the interference between the $p=0$ external direct reflection and the $p=2$ refractive rainbow rays with one internal reflection [4]. However it has been considered that in the nuclear rainbow, which is caused by a Luneberg lens [10] of a nuclear potential $(p=1)$ with a smoothly diffused surface, direct reflection scarcely occurs. This is seen in Fig. 3(b) where no high-frequency oscillations appear in the calculations using the real and imaginary potentials with a smooth surface. On the other hand, we see in Fig. 3(c) that the high-frequency oscillations are created by the interference between the reflective nearside component caused by the sharp-edged imaginary potential and the farside component. Thus the high-frequency oscillations superimposed on the Airy structure are considered to be the nuclear ripples because they have the same physical origin as those of the meteorological rainbow. In Fig. 3(a) it is found that the coupling to the excited states also contributes in creating the reflective nearside waves that are caused by 


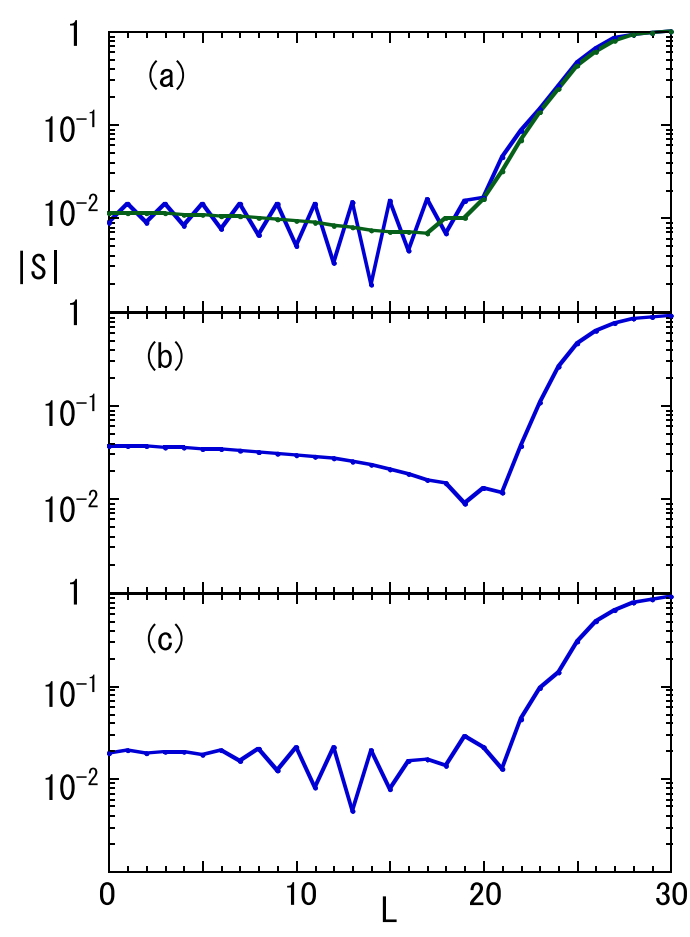

FIG. 5. (Color online) The moduli of the calculated $S$ matrices in Figs. 3(a), 3(b), and 3(c). The lines are explained in the caption of Fig. 3.

the sharp-edged imaginary potential in Fig. 3(c). The nearside scattering waves that are responsible for the generation of the high-frequency oscillations in Figs. 3(a) and 3(c) correspond to the externally reflected waves in Mie scattering of the meteorological rainbow. Now the physical meaning and the origin of the extremely thin-skinned volume-type imaginary potential needed in Refs. [18,25] are clear. They were needed to mimic the effect of the channel coupling to enhance the reflective waves.

In the single channel calculations, the increase of the nearside component that corresponds to the externally reflective waves is only attained by using the extraordinary small diffuseness parameter $a_{W}=0.1$ for the imaginary potential. This necessarily accompanies introducing the additional surface imaginary potential at the large radius to preserve the net absorption, i.e., the volume integral of the imaginary potential. In the coupled channel calculations in Fig. 3(a), in fact, no surface imaginary potential at the large radius was needed. It is important to treat channel coupling to the excited states microscopically to avoid the unphysically sharp-edged volume-type imaginary potential and the surface imaginary potential at the large radius.

How the ripple structure emerges on the Airy structure by the reflective waves is shown in Fig. 6 where the angular distributions-calculated by replacing the $S_{L}$ matrices with the orbital angular momentum $L=0 \sim L_{c}$ generated by the potential of Fig. 3(b) by those generated by the potential of Fig. 3(c)—are displayed. With around $L_{c}=8$ (corresponding impact parameter $b=2.1 \mathrm{fm}$ ) the ripple structure starts to emerge on the $A 1$ Airy peak in the backward angles beyond $\theta \approx 100^{\circ}$ and with around $L_{c}=14(b=3.6 \mathrm{fm})$ they also

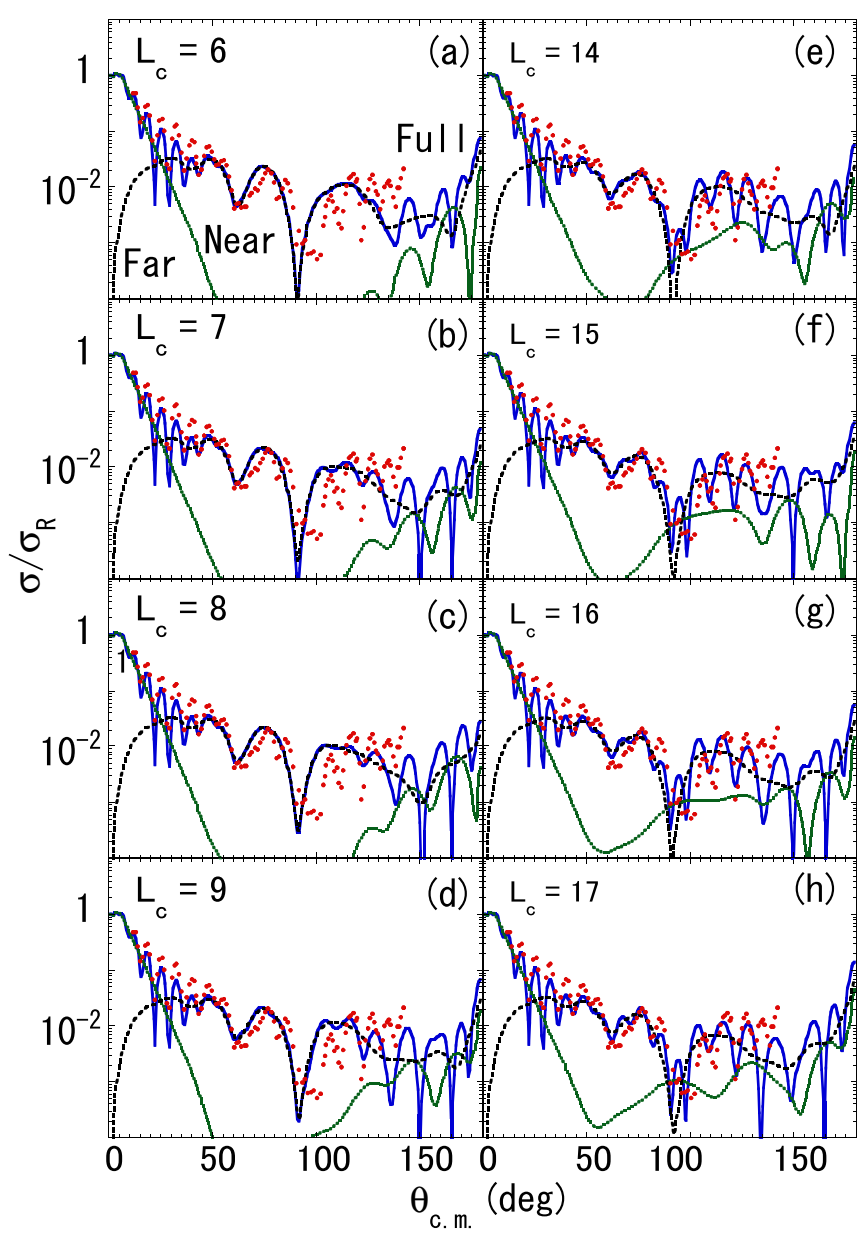

FIG. 6. (Color online) The angular distributions in ${ }^{16} \mathrm{O}+{ }^{12} \mathrm{C}$ scattering at $E_{L}=115.9 \mathrm{MeV}$ calculated by replacing the $S_{L}$ with $L=0 \sim L_{c}$ among the $S_{L}$-matrices generated by the potential used for Fig. 3(b) by those generated by the potential used for Fig. 3(c) (solid line) and its farside (dashed line) and nearside (dotted line) components are compared with the experimental data (points) [23].

appear on the Airy peak $A 2$ in the intermediate angles before $\theta \approx 90^{\circ}$. For $L_{c}=14-17(b=3.6-4.3 \mathrm{fm})$, which corresponds to the radius of the sharp edge of the imaginary potential in Fig. 4 (black dashed line), the high-frequency oscillations in phase with the experimental data are reproduced. The impact parameters of these partial waves are significantly smaller than $b=5.9 \mathrm{fm}$ of the grazing partial wave $L=24$ for which $\left|S_{L}\right|=0.5$ (see Fig. 5). The channel coupling in Fig. 3(a) plays two roles; that is, enhancing the reflective waves and enhancing absorption at the surface, which makes it possible to use the relatively larger diffuseness parameter $a_{W}=0.2 \mathrm{fm}$ and no surface imaginary potential needed in Fig. 3(c).

Finally we mention that the high-frequency oscillations are not due to the elastic transfer of the $\alpha$ particle [31]. In fact, we see in the detailed coupled reaction channels calculations of ${ }^{16} \mathrm{O}+{ }^{12} \mathrm{C}$ scattering at $E_{L}=115.9 \mathrm{MeV}$ in Ref. [33] that the contribution of the elastic transfer is three orders of magnitude smaller than the experimental data. Also the present calculations take into account the one-nucleon exchange effect, which is suggested to prevail over other transfer reactions [33], 
by using the effective interaction DDM3Y, in which the knock-on exchange effect is incorporated $[14,30]$.

To summarize, we have calculated ${ }^{16} \mathrm{O}+{ }^{12} \mathrm{C}$ scattering with the Airy structure at $E_{L}=115.9 \mathrm{MeV}$ using a coupled channels method with an extended double folding (EDF) potential that is derived by using the microscopic realistic wave functions for ${ }^{12} \mathrm{C}$ and ${ }^{16} \mathrm{O}$ by taking account of excited states of the $2^{+}(4.44 \mathrm{MeV})$ and $3^{-}(9.64 \mathrm{MeV})$ states of ${ }^{12} \mathrm{C}$ and the $3^{-}(6.13 \mathrm{MeV})$ and $2^{+}(6.92 \mathrm{MeV})$ states of ${ }^{16} \mathrm{O}$. Our calculations reproduce the high-frequency oscillations superimposed on the Airy structure. It is found that the high-frequency oscillations are nothing but the nuclear ripples similar to those superimposed on the Airy structure in Mie scattering of the meteorological rainbow. The nuclear ripples are generated by the interference between the refractive waves and the externally reflected waves. The coupling to the excited states of ${ }^{16} \mathrm{O}$ and ${ }^{12} \mathrm{C}$ plays the role of creating external reflection. Although the active interactions in the nuclear and the meteorological rainbows are very different, we see the similarity in that both have the ripple structure on the Airy structure due to the same origin of the interference between refractive waves and the externally reflected waves. It is startling that a classical concept of a ripple in the meteorological rainbow persists in the quantum nuclear rainbow.

One of the authors (S.O.) thanks the Yukawa Institute for Theoretical Physics for the hospitality extended during a stay in Spring 2014. Part of this work was supported by the Grant-in-Aid for the Global COE Program "The Next Generation of Physics, Spun from Universality and Emergence" from the Ministry of Education, Culture, Sports, Science and Technology (MEXT) of Japan.
[1] R. Descartes, Le Discours de la methode (sous-titre Pour bien conduire sa raison, et chercher la verité dans les sciences) plus la Dioptrique, Les Meteores et la Geometrie (Leiden, 1637).

[2] I. Newton, Opticks or, a Treatise of the Reflexions, Refractions, Inflexions and Colours of Light (London, 1704); (Dover, New York, 1952).

[3] G. B. Airy, Trans. Camb. Philos. Soc. 6, 379 (1838).

[4] H. M. Nussenzveig, Sci. Am. 236, 116 (1977); Diffraction Effects in Semiclassical Scattering (Cambridge University Press, Cambridge, 1992).

[5] K. W. Ford and J. A. Wheeler, Ann. Phys. 7, 259 (1959).

[6] D. A. Goldberg and S. M. Smith, Phys. Rev. Lett. 29, 500 (1972); D. A. Goldberg, S. M. Smith, H. G. Pugh, P. G. Roos, and N. S. Wall, Phys. Rev. C 7, 1938 (1973); D. A. Goldberg, S. M. Smith, and G. F. Burdzik, ibid. 10, 1362 (1974).

[7] J. N. L. Connor and D. Farrelly, J. Chem. Phys. 75, 2831 (1981).

[8] G. Ziegler, M. Rädle, O. Pütz, K. Jung, H. Ehrhardt, and K. Bergmann, Phys. Rev. Lett. 58, 2642 (1987).

[9] A. W. Kleyn and T. C. M. Horn, Phys. Rep. 199, 191 (1991); C. O. Reinhold, J. Burgdörfer, K. Kimura, and M.-h. Mannami, Phys. Rev. Lett. 73, 2508 (1994).

[10] F. Michel, G. Reidemeister, and S. Ohkubo, Phys. Rev. Lett. 89, 152701 (2002).

[11] R. L. Lee, Jr., Appl. Opt. 37, 1506 (1998).

[12] J. P. A. J. van Beeck and M. L. Riethmuller, Appl. Opt. 35, 2259 (1996).

[13] D. T. Khoa, W. von Oertzen, H. G. Bohlen, and S. Ohkubo, J. Phys. G 34, R111 (2007), and references therein.

[14] M. E. Brandan and G. R. Satchler, Phys. Rep. 285, 143 (1997).
[15] F. Michel, S. Ohkubo, and G. Reidemeister, Prog. Theor. Phys. Suppl. 132, 7 (1998).

[16] S. Ohkubo, T. Yamaya, and P. E. Hodgson, Nuclear clusters. in Nucleon-Hadron Many-Body Systems, edited by H. Ejiri and H. Toki (Oxford University Press, Oxford, 1999), p. 150.

[17] D. T. Khoa, W. von Oertzen, H. G. Bohlen, and F. Nuoffer, Nucl. Phys. A 672, 387 (2000).

[18] M. P. Nicoli et al., Phys. Rev. C 60, 064608 (1999).

[19] S. Ohkubo and K. Yamashita, Phys. Rev. C 66, 021301(R) (2002); S. Ohkubo, Acta Phys. Hung. A 18, 287 (2003).

[20] M. E. Brandan et al., Phys. Rev. C 34, 1484 (1986).

[21] D. T. Khoa, W. von Oertzen, and H. G. Bohlen, Phys. Rev. C 49, 1652 (1994).

[22] A. A. Ogloblin et al., Phys. Rev. C 57, 1797 (1998).

[23] M. P. Nicoli et al., Phys. Rev. C 61, 034609 (2000).

[24] A. A. Ogloblin et al., Phys. Rev. C 62, 044601 (2000).

[25] S. Szilner et al., Phys. Rev. C 64, 064614 (2001).

[26] S. Ohkubo and K. Yamashita, Phys. Lett. B 578, 304 (2004).

[27] S. Okabe, Tours Symposium on Nuclear Physics II, edited by H. Utsunomiya et al. (World Scientific, Singapore, 1995), p. 112.

[28] Y. Suzuki, Prog. Theor. Phys. 55, 1751 (1976); 56, 111 (1976).

[29] M. Kamimura, Nucl. Phys. A 351, 456 (1981).

[30] A. M. Kobos, B. A. Brown, P. E. Hodgson, G. R. Satchler, and A. Budzanowski, Nucl. Phys. A 384, 65 (1982); A. M. Kobos, B. A. Brown, R. Lindsaym, and G. R. Satchler, ibid. 425, 205 (1984).

[31] S. Szilner, W. von Oertzen, Z. Basrak, F. Haas, and M. Milin, Eur. Phys. J. A 13, 273 (2002).

[32] S. Ohkubo and Y. Hirabayashi, Phys. Rev. C 89, 051601(R) (2014).

[33] A. T. Rudchik et al., Eur. Phys. J. A 44, 221 (2010). 